Key Engineering Materials Vols. $192-195$ (2001) pp. 243-246

online at http://www. scientific.net

(C) (2001) Trans Tech Publications, Switzerland

(C) 2001 Trans Tech Publications, Switzerland

\title{
Emulsion Crosslinking as a New Manufacturing Route to Produce Hydroxylapatite Particulates in a Network of Starch
}

\author{
P.B. Malafaya, F. Stappers and R.L. Reis \\ Department of Polymer Engineering, University of Minho, Campus de Gualtar, \\ PT-4710-057 Braga, Portugal
}

Keywords: Bioactive, Calcium Phosphate, Carrier, Fillers, Particulate, Reinforcement

\begin{abstract}
:
Bioactive particulates with tailored resorption rates can be very useful on biomedical applications such as filling of bone and dentristy defects, carriers for controlled release of bioactive agents and reinforcement in polymer/bioceramic orthopaedic composites. The aim of the present study was to develop a new processing route to produce composite particulates based on hydroxylapatite (HA) and starch. The newly proposed processing route consists on the crosslinking of starch that is able to encapsulate the HA particles creating a composite material with different sizes (from 3000-700 $\mu \mathrm{m})$ which is resorbable and bioactive. This work also involves the characterization of the developed material, as well as the study of the in-vitro degradation and bioactive behaviour of the particulates. The particulate biomaterials have shown to be bioactive even after small immersion periods on a simulated body fluid (SBF). The obtained results (in-vitro) indicate that particulates will be expected to be resorbed when implanted. The developed materials present an interesting combination of morphological and chemical properties that make them potential biomaterials to be used as fillers of bone defects, drug delivery carriers or as reinforcement of bioactive starch-based composites.
\end{abstract}

\section{Introduction}

Particulates can be best described as relative small particles available in a variety of size fractions.[1] Hydroxylapatite (HA) has been used as spacer in several shapes. As this material bonds chemically to the bony tissue or is resorbed by the organism, a loosening of the spacer never occurs and the patient does not suffer pain problems.[2] In fact, the main interest of calcium phosphate materials comes from the fact that, from a chemical point of view, they are close the mineral part of calcified tissues (bone, teeth). The structure of this mineral part is always represented as an idealized hydroxylapatite (HA).[3] It exists a real similarity between the chemical composition of synthetic calcium phosphate biomaterials and bony tissues which is surely in close connection with their well-known biocompatibility.[4] Moreover, this analogy induces another property which increases their superiority compared with other bioceramics - they are bioactive and either their resorption never causes particularly unfavourable reactions with the surrounding tissues or the biomaterial remains in the local and is able to bond to bony tissue.[4] Bioactivity, biocompatibility and osteogenicity explain the great current interest on developing adequate calcium-phosphate biomaterials. These materials can be produced in a variety of forms including particulates, sintered block forms, porous scaffolds or fibres. Choosing among these forms will depend upon the end-use application. In this study, a new manufacturing route to produce composite particulates was developed. The materials are based on HA due to its osteo-related properties and on starch that already proved to be biocompatible and able to be used in several 
applications in the biomedical field. [5,6] The developed particulates present very promising properties that make them a potential biomaterial to be applied as filler of bone (dentristy) defects, as drug delivery carriers or even as a reinforcement phase to produce bioactive polymeric matrix composites.

\section{Materials \& Methods}

The developed methodology is based on crosslinking a starch powder that will encapsulate the hydroxylapatite (HA) particles. An emulsion crosslinking method has been applied, consisting on dissolving the modified starch (Paselli (II), The Netherlands), HA (supplied by Plasma Biotal, UK) and crosslinking agent (TriSodium TrimetaPhosphate-TSTP-from Sigma-Aldrich) in a water-phase. The crosslinking reaction is started by adding a basic substance $(\mathrm{NaOH})$ to the solution. The emulsion is obtained by mixing a oilic phase (paraffin wax, from Fluka) with the water phase and using a suitable non-ionic surfactant to stabilise the W/O emulsion. The phases are separated after the particles have been formed, washed several times and then dried in an oven at $60^{\circ} \mathrm{C}$. The final composite particles are then obtained.

Sieving and light scattering analysis were used to classify the particle sizes and respective distributions. The particles were further characterised morphologically by scanning electron microscopy (SEM) and structurally by X-ray diffraction (XRD) in order to identify the crystalline phases present in the particulates. The particulates degradation behaviour was evaluated after several pre-fixed periods (up to 30 days) in an isotonic saline solution $(\mathrm{NaCl} 0.154 \mathrm{M}, \mathrm{pH}=7.40)$ as a function of the respective particle size. The $\mathrm{pH}$ was also continuously monotorised. The evolution of the elemental concentration of $\mathrm{Ca}, \mathrm{P}$ and $\mathrm{Na}$ was accessed by induced coupled plasma (ICP) spectroscopy. Bioactivity assays were carried out in a simulated body fluid ( $\mathrm{SBF}$ ), at $37^{\circ} \mathrm{C}, \mathrm{pH}=$ 7.25 , for several immersion periods up to 30 days. The apatite-like layer formed was characterized morphologically by SEM and the evolution of $\mathrm{Ca}$ and $\mathrm{P}$ concentrations was follow along time by ICP spectroscopy.

\section{Results \& Discussion}

The developed emulsion crosslinking method made possible to obtain composite particles, approximately spherical, with diameters ranging from $3000 \mu \mathrm{m}$ to $700 \mu \mathrm{m}$ with typical morphologies as shown in Figure 1 (a) and (b). By SEM observation it is possible to see the starch phase involving the HA particles forming a composite particulate. The obtained particles were classified according to their mean diameter in: (1) $3500 \mu \mathrm{m}$; (2) $2500 \mu \mathrm{m}$; (3) $1500 \mu \mathrm{m}$ and (4) 700 $\mu \mathrm{m}$. These will be the designation used when referring to the distinct classes of particles.

XRD analysis confirmed that the composite particulates were composed mainly by pure HA since an almost perfect match with the standard HA JCPDS 9-432 pattern was obtained. The starch phase present in the composite particulates is not identified by XRD spectra due to the much stronger signal emanated by the crystalline ceramic phase. The observed decrease on the HA peaks intensity is due to the presence of a certain amount of polymer within the particulates.
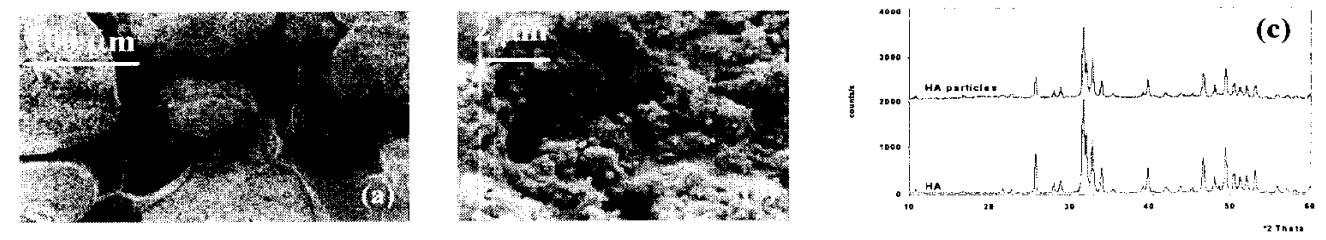

Fig. 1 - Characterization of HA particles: (a) SEM micrographs showing the network of starch involving the HA particles and (b) HA entrapped in the biodegradable polymeric matrix; (c) XRD spectra of HA used as raw material and of the $\mathrm{HA} /$ starch particulates. 
The degradation kinetics was faster in the particles with smaller diameter due to the higher relative surface area (see fig. 2 a). A non-sintered HA sample (obtained from Plasma Biotal, UK, with a mean particle size of $3 \mu \mathrm{m}$ ) was used as a control. The faster degradation of the composite particles (as compared to the control), that might lead to a higher in-vivo resorbability, is due to their final composition and structure after the emulsion crosslinking processing route. In fact, the results seem to indicate that this processing technique may constitute an alternative route for designing particulate ceramic materials, which might be advantageous for several applications where bioactivity and resorbability are needed. The degradation products originated from these particulates not only are not expected to disturb the metabolism of the organism, but in fact they might contribute to its growth since they are the basic components of its inorganic part. [7] The starch-based polymeric part is also biodegradable, meaning that it will degrade chemically with chain breakage and leaching of molecular chains as reported before [8] for injection moulded starch-based polymers. These polymers will degrade in-vivo to small molecular weight compounds that are either metabolized or excreted, obviating the need for its removal. A range of starch-based polymers has already proved to be suitable to several applications in the biomedical field. $[5,9]$ The final composition of the composite particulate including the presence of the starch phase leads to a decrease in the pH vs. time values when compared to the pure HA control (see fig. 2 b). When particulates are implanted, instead of bulk materials, the ion diffusion increases and it can change the local $\mathrm{pH}$. There is a possibility, if the degradation rate is too high that the $\mathrm{pH}$ will exceed the minimum physiological value, slowing down the bony tissue growth. [7] It is possible to observe in Figure 2 (a) that even the maximum weight loss of the particulates with the lower mean particle size is stabilised (around 12\%) after a 30 days degradation period. The minimal achieved $\mathrm{pH}$ value is around 5. It is expected that the $\mathrm{pH}$ decreased will be much less accentuated in the body due to the continuous circulation of the buffered body fluids. So, although in-vivo studies are required to draw some conclusions, it seems that no problems are to be foreseen due to the resorbability of these particulates.
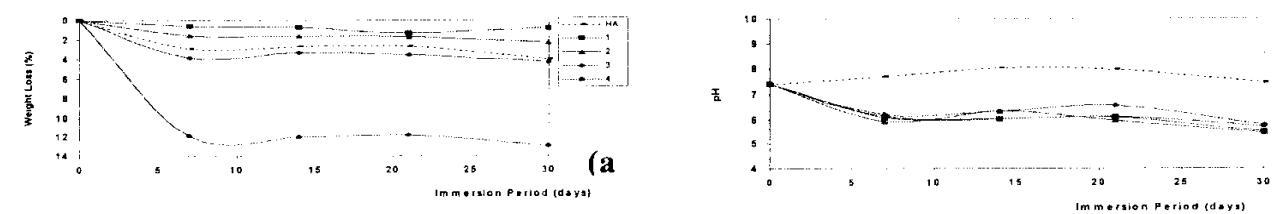

Fig. 2 - Degradation behaviour of the HA particles evaluated by (a) weight loss and (b) the evolution of the pH of the degradation solution. (-: HA; : $1 ;: 2 ; \bullet: 3 ; \mathbf{A}: 4)$
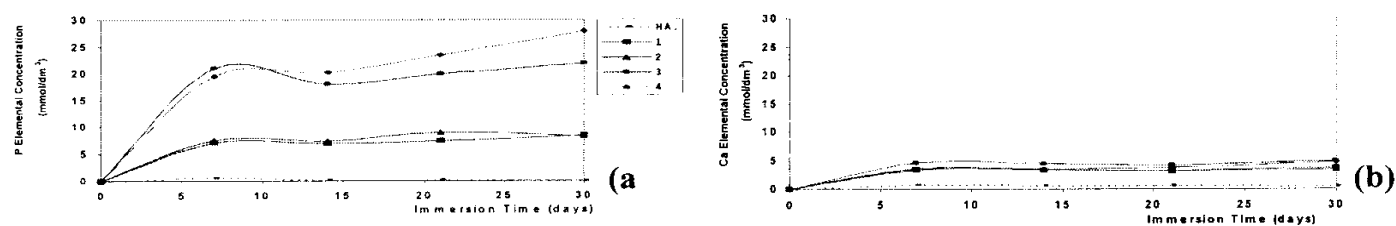

Fig. 3 - Evolution of (a) $\mathrm{P}$ and (b) Ca concentrations as function of the mean diameter of the HA particles in the degradation assays. (-: HA; $\mathbf{0}: 1 ; \bullet: 2 ; 0: 3 ; \mathbf{A}: 4)$

By ICP analyses it was possible to follow the evolution of the $\mathrm{Ca}$ and $\mathrm{P}$ concentrations during the degradation assays. The release of $P$ is a function of particle size increasing with the surface area, i.e. it is observed a higher release of $\mathrm{P}$ for smaller particles sizes. For $\mathrm{Ca}$, there is a release in the initial degradation period that tends to stabilise after a 7 days period. These results indicate that in a isotonic saline solution there is only a dissolution of the particulates, and not any dissolutionreprecipitation phenomena. 
A much smaller release of $P$ was found on tests carried out in SBF (see fig. 4). This seems to indicate a dissolution and reprecipitation phenomena that is responsible for the formation of the surface layers observed in Figure 5
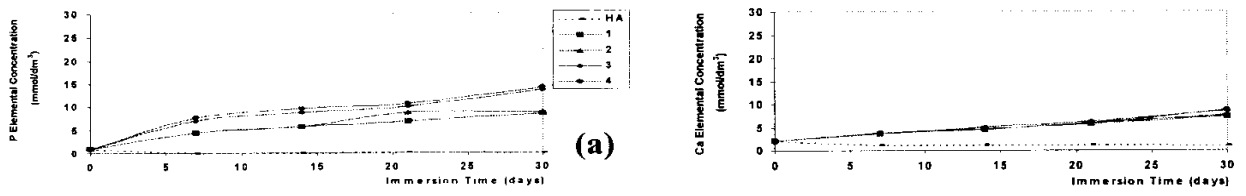

Fig. 4 - Evolution of (a) $\mathrm{P}$ and (b) $\mathrm{Ca}$ concentrations as function of the mean diameter of the HA particles in the bioactivity assays (ICP results). (-: HA; $\mathbf{\mathbf { n }}: 1 ; \bullet: 2 ; 0: 3 ; \mathbf{\Delta}: 4)$

In fact, on Figure 5, it is possible to observe the formation of a dense and uniform apatite-like layer that was produced after 14 days immersion in SBF. The so-called cauliflower morphology [10] was found in the surface of the particulates (independently of the particle size) as shown in Figure 5. These observations indicate an in-vitro bioactive behaviour of the particulates.
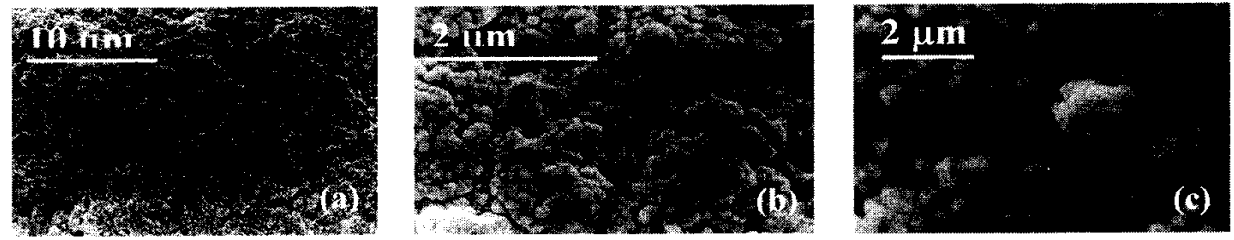

Fig. 5 - SEM micrographs of HA particles after 14 days of immersion in SBF showing the typical morphology of the apatite-like layer formed in the surface of the particles.

\section{Conclusions}

The developed particulates present morphological, structural, degradation and bioactive properties that might be very interesting for several biomedical applications, namely for filling bone (dentristry) defects, or to be used as carriers for the controlled release of drugs or other bioactive agents. The advantage of the proposed processing route is that these agents may be incorporated in the particles during the manufacturing route, instead of just being adsorbed on their surface. Another potential application that is being assessed is the use of the particulates for the reinforcement of polymeric starch-based degradable matrices. This will eventually lead to an improved bioceramic/polymer interfacial adhesion (due to the presence of starch on the "ceramic" phase) originating composites with an enhanced mechanical behaviour to be used on bone replacement and fixation applications.

\section{References}

[1] JE Lemons, PK Bajpai, et al, in Bioceramics: Materials Characteristics versus in vivo Behaviour, ed: P. Ducheyne, JE Lemons, Annals of the New York Academy of Sciences, vol. 523 (1988) 278

[2] H Oonishi, in Biomaterials-Hard Tissue Repair \& Replacement, ed: D. Muster, Elsevier Sci Pub, Amesterdam (1992) 17

[3] JL Lacout, in Biomaterials-Hard Tissue Repair \& Replacement, ed: D. Muster, Elsevier Sci Pub, Amesterdam (1992) 81

[4] G Grimandi, P Weiss, F Millot, G Daculsi, J Biomed Mat Res, 39 (1998) 660

[5] SC Mendes, Y Bovell, RL Reis, CA Bltterswijk, $25^{\text {th }}$ Annual Meeting of Society for Biomaterials, San Diego, USA (1998) 9

[6] CS Pereira, AM Cunha, RL Reis, B Vazquez, JS Román, J Mat Sci: Mat in Med, 9 (1998) 825

[7] AM Gatti, D Zaffe, in Biomaterials - Hard Tissue Repair \& Replacement, ed: D. Muster, Elsevier Sci Pub, Amesterdam (1992) 97

[8] RL Reis, SC Mendes, AM Cunha, MJ Bevis, Polymer International, 43 (1997) 347

[9] ME Gomes, AS Ribeiro, PB Malafaya. RL Reis, AM Cunha, Biomaterials (2000) In Press

[10] RL Reis, AM Cunha, MH Fernades, RN Correia, J Mater Sci: Mat in Med, 8 (1997) 897 